medRxiv preprint doi: https://doi.org/10.1101/2021.10.29.21265645; this version posted October 30, 2021. The copyright holder for this preprint (which was not certified by peer review) is the author/funder, who has granted medRxiv a license to display the preprint in

All rights reserved. No reuse allowed without permission.

\title{
Deep neural networks learn general and clinically relevant representations of the ageing
} brain

Esten H. Leonardsen ${ }^{1,2,}{ }^{*}$, Han Peng ${ }^{3}$, Tobias Kaufmann ${ }^{2,4}$, Ingrid Agartz ${ }^{2,5,6}$, Ole A. Andreassen ${ }^{2}$, Elisabeth Gulowsen Celius ${ }^{7,8}$, Thomas Espeseth ${ }^{1,9}$, Hanne F. Harbo ${ }^{7,8}$, Einar A. Høgestøl $1^{1,2,7}$, Ann-Marie de Lange ${ }^{1,10,11}$, Andre F. Marquand ${ }^{12}$, Didac Vidal-Piñeiro ${ }^{1}$, James M. Roe ${ }^{1}$, Geir Selbæk ${ }^{13,14}$, Øystein Sørensen ${ }^{1}$, Stephen M. Smith ${ }^{3}$, Lars T. Westlye ${ }^{1,2, x, \#}$, Thomas Wolfers ${ }^{1,2, \#,}$ Yunpeng Wang ${ }^{1, \#}$ \footnotetext{
University of Oslo, Oslo, Norway Services, Stockholm County Council, Stockholm, Sweden

Lausanne, Switzerland

\# equal contribution

* Corresponding author

Esten Leonardsen (estenhl@psykologi.uio.no)

Department of Psychology, University of Oslo,

Postboks 1094 Blindern, 0317 OSLO, Norway,

$+4722845000$
}

1 Department of Psychology, University of Oslo, Oslo, Norway

2 Norwegian Centre for Mental Disorders Research (NORMENT), Oslo University Hospital \& Institute of Clinical Medicine,

3 Wellcome Centre for Integrative Neuroimaging (WIN FMRIB), University of Oxford, Oxford, OX3 9DU, United Kingdom 4 Department of Psychiatry and Psychotherapy, Tübingen Center for Mental Health, University of Tübingen, Germany 5 Department of Psychiatric Research, Diakonhjemmet Hospital, Oslo, Norway

6 Centre for Psychiatry Research, Department of Clinical Neuroscience, Karolinska Institutet \& Stockholm Health Care

7 Department of Neurology, Oslo University Hospital, Norway

8 Institute of Clinical Medicine, University of Oslo, Oslo, Norway

9 Department of Psychology, Bjørknes University College, Oslo, Norway

10 LREN, Centre for Research in Neurosciences-Department of Clinical Neurosciences, CHUV and University of Lausanne,

11 Department of Psychiatry, University of Oxford, Oxford, UK

12 Donders Institute for Brain, Cognition and Behaviour, Radboud University Medical Centre, Nijmegen, Netherlands

13 Norwegian National Advisory Unit on Aging and Health, Vestfold Hospital Trust, Tønsberg, Norway

14 Department of Geriatric Medicine, Oslo University Hospital, Oslo, Norway

X KG Jebsen Center for Neurodevelopmental Disorders, University of Oslo, Oslo, Norway

NOTE: This preprint reports new research that has not been certified by peer review and should not be used to guide clinical practice. 
medRxiv preprint doi: https://doi.org/10.1101/2021.10.29.21265645; this version posted October 30, 2021. The copyright holder for this preprint (which was not certified by peer review) is the author/funder, who has granted medRxiv a license to display the preprint in All rights reserved. No reuse allowed without permission.

\section{Abstract}

The discrepancy between chronological age and the apparent age of the brain based on neuroimaging data - the brain age delta - has emerged as a reliable marker of brain health. With an increasing wealth of data, approaches to tackle heterogeneity in data acquisition are vital. To this end, we compiled raw structural magnetic resonance images into one of the largest and most diverse datasets assembled $(\mathrm{n}=53542)$, and trained convolutional neural networks (CNNs) to predict age. We achieved state-of-the-art performance on unseen data from unknown scanners $(\mathrm{n}=2553)$, and showed that higher brain age delta is associated with diabetes, alcohol intake and smoking. Using transfer learning, the intermediate representations learned by our model complemented and partly outperformed brain age delta in predicting common brain disorders. Our work shows we can achieve generalizable and biologically plausible brain age predictions using CNNs trained on heterogeneous datasets, and transfer them to clinical use cases. 
medRxiv preprint doi: https://doi.org/10.1101/2021.10.29.21265645; this version posted October 30, 2021. The copyright holder for this preprint (which was not certified by peer review) is the author/funder, who has granted medRxiv a license to display the preprint in

All rights reserved. No reuse allowed without permission.

\section{Introduction}

Neurodevelopmental and age-related changes in the brain play a crucial role in the etiology of complex neurological ${ }^{1}$ and mental disorders ${ }^{2}$. Predictive models for an individuals age based on magnetic resonance imaging (MRI) have been used to estimate normative trajectories across the lifespan ${ }^{3-6}$. Individual deviations from these trajectories, often called the brain age delta, have been linked to brain health ${ }^{7,8}$, and more extreme deviations observed in patients with schizophrenia $(\mathrm{SCZ})^{9-11}$, depression ${ }^{12}$, cognitive impairment ${ }^{13,14}$, dementia ${ }^{15}$, Alzheimer's disease $(\mathrm{AD})^{16}$ and multiple sclerosis $(\mathrm{MS})^{17}$, implying that such deviations could be a feasible biological marker for various brain disorders.

The brain age of an individual is typically estimated from brain images using statistical learning techniques. The first-generation models were relatively simple, typically based on independent vox$e^{3}{ }^{3}$ or a limited number of imaging-derived phenotypes (IDPs) reflecting brain properties such as volumetric measures of different regions ${ }^{18}$. These models generally estimated linear relationships, were restricted in scale, and were trained on datasets with tens, hundreds or a few thousand participants $^{7}$. In parallel with continuous computational advances the exponential growth of MRI data has enabled deep learning models of scale for accurate brain age estimation ${ }^{6,19}$. Deep learning models can take minimally- or non-preprocessed 3-D images as input - avoiding computationally demanding and hypothesis-driven ${ }^{20}$ choices during image processing - and model complex non-linear relations between voxels. One such model is the Simple Fully Convolutional Network (SFCN), a novel deep Convolutional Neural Network (CNN) that won the Predictive Analysis Challenge for brain age prediction in 2019 (PAC2019) ${ }^{21,22}$. While such deep learning approaches allow for the prediction of brain age with unprecedented accuracy, and can potentially help us identify idiosyncratic regional patterns of neurodevelopment and ageing at the individual level ${ }^{23,24}$, their complexity also comes with a risk of overfitting, namely finding patterns in the training data which do not generalize well to new, previously unseen, participants ${ }^{25}$.

Transfer learning, a deep learning technique widely used in other areas of applied machine learning research, has recently gained momentum in neuroimaging ${ }^{26}$. Here, learned intermediate representations can be shared between tasks, allowing a model to be transferred to a problem or a dataset it was initially not trained for ${ }^{27}$. This approach has arguably been one of the core developments underlying the practical success of deep learning in a range of computer vision problems ${ }^{28}$ by using models trained on general purpose datasets, typically ImageNet ${ }^{29}$, and fine-tuning them towards a wide array of tasks. Recent studies have shown that transfer learning yields promising results also 
medRxiv preprint doi: https://doi.org/10.1101/2021.10.29.21265645; this version posted October 30, 2021. The copyright holder for this

preprint (which was not certified by peer review) is the author/funder, who has granted medRxiv a license to display the preprint in

All rights reserved. No reuse allowed without permission.

for brain age predictions ${ }^{30}$ and clinical classifications based on MRI data ${ }^{31,32}$. This exemplifies the need for robust, pretrained models on massive multisite datasets, that can be translated to smaller clinical samples, and, ultimately, to individual cases in a clinical setting. Modelling brain age as a pre-training step has obvious advantages as age is a variable which is available in most current MRI datasets. Additionally, the representations learned by the brain age models, representing partly independent dimensions of age-related variance, could be of direct importance in individual level brain phenotyping.

In the present study we trained deep neural network models for brain age prediction on structural MRI data from 53542 healthy individuals between 3 and 95 years of age to test their ability to generalize, and demonstrate the downstream applicability and biological relevance of a properly generalizing model. We used the Simple Fully Convolutional Network with a softmax output (SFCN-sm) which predicts age as a discrete probability distribution, and compared its accuracy and generalizability with two proposed variants of the architecture; a regression variant (SFCN-reg), directly predicting continuous age, and a ranking variant (SFCN-rank) encoding age as an ordinal vector. Brain age delta was computed as the difference between the predicted and chronological age. To estimate the sensitivity and clinical relevance of our best model, we tested for associations between brain age delta and a range of clinical and biological phenotypes, and sociodemographic and lifestyle variables, in unseen data from a population sample. To further demonstrate the applicability of the model, we employed the pretrained SFCN-reg in a transfer learning context to predict case-control status for AD, MS, mild cognitive impairment (MCI), SCZ, mood disorders and psychotic disorders on datasets obtained from a range of MRI scanners. To promote transparency and reproducibility we have implemented an easy-to-use Keras interface for all the trained models, both the brain age and clinical predictors, and a pipeline for preprocessing images, available on our GitHub http://www.github.com/estenhl/pyment-public.

\section{Results}

We compiled 21 publicly available datasets with T1-weighted MRI scans into a large and diverse imaging dataset (total $\mathrm{N}=53542$; female $\mathrm{N}=27715$; age range=3-95), and trained a Simple Fully Convolutional 3-dimensional CNN with 6 convolutional blocks and a softmax output layer (SFCNsm, Figure 1b), as introduced in Peng et $\mathrm{al}^{21}$. We then proposed two alternatives for the prediction layer of this architecture, the first based on regression (SFCN-reg) and the second based on ranking ${ }^{33}$ (SFCN-rank) (see Methods for details). Due to the time-consuming process of model evaluation, 
medRxiv preprint doi: https://doi.org/10.1101/2021.10.29.21265645; this version posted October 30, 2021. The copyright holder for this preprint (which was not certified by peer review) is the author/funder, who has granted medRxiv a license to display the preprint in perpetuity.

All rights reserved. No reuse allowed without permission.

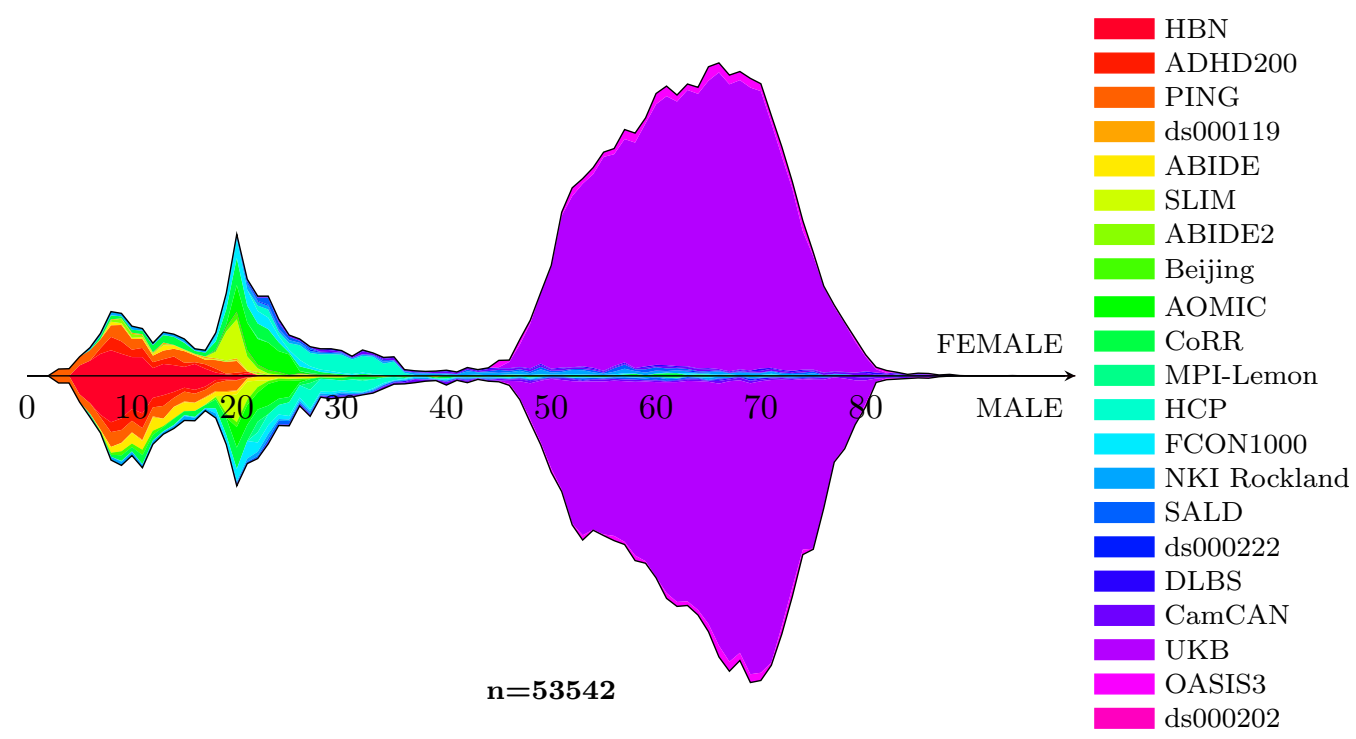

(a) Age distribution of the reference dataset

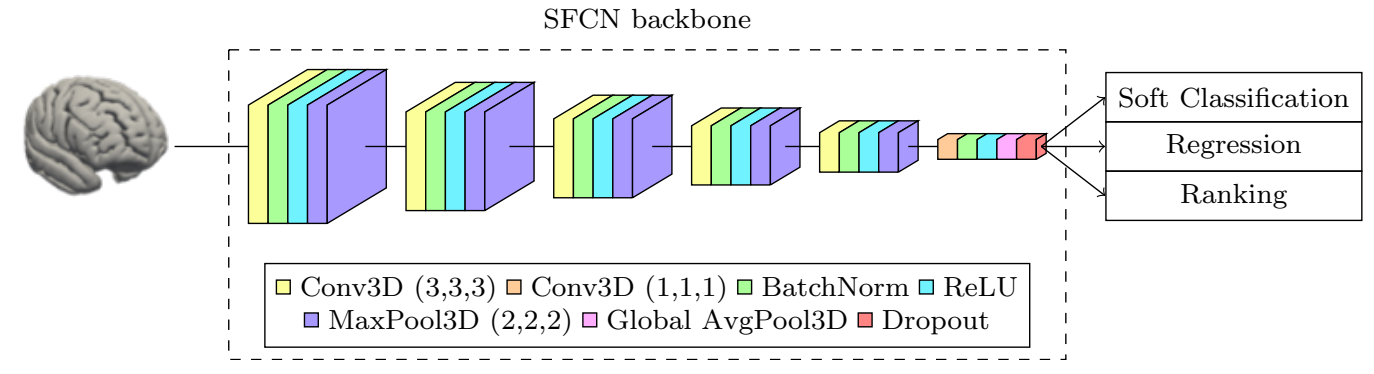

(b) Brain age model architecture

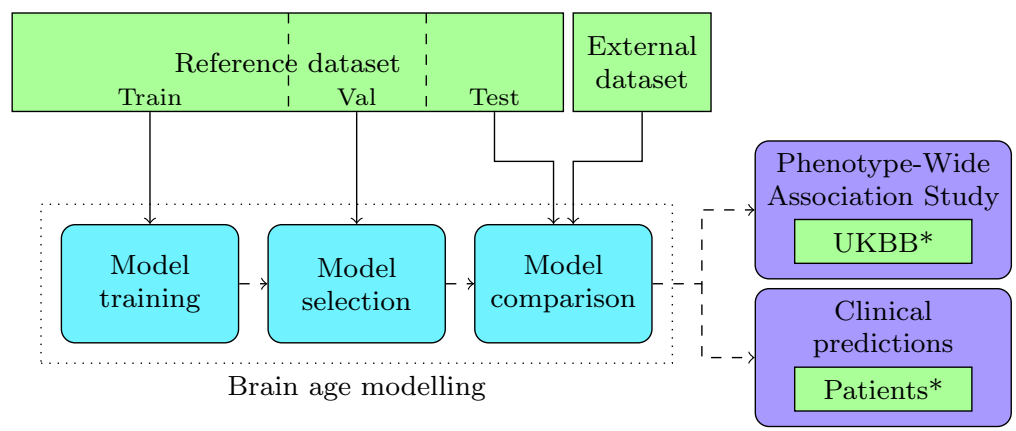

(c) Flowchart of the modelling process and applications

Figure 1: An overview of the dataset and models used for brain age modelling. (a) The reference dataset contains 53542 healthy participants from 21 datasets, with ages ranging from 3 to 95 years. For both sexes

the age distribution is approximately bimodal, with the first large group of participants from 5 to 35 years originating from multiple studies, and the second from 45 to 80 mostly consisting of participants from the UKBB.

(b) We implemented three model variants for predicting brain age, all based on the contest-winning SFCN architecture $^{21}$. The architectures of the three variants differ only in the final prediction layer. All the models take minimally preprocessed T1-weighted MRI images as input. (c) The modelling process consisted of three steps, each utilizing a different portion of the reference dataset. In the final comparison we also employed an external dataset, coming from previously unseen scanners, to assess the generalization capabilities of the different models. Having found the best brain age model, we subsequently applied it in two applications: A phenome-wide association study (PheWAS), and a case-control comparison including several clinical conditions. 
medRxiv preprint doi: https://doi.org/10.1101/2021.10.29.21265645; this version posted October 30, 2021. The copyright holder for this preprint (which was not certified by peer review) is the author/funder, who has granted medRxiv a license to display the preprint in

All rights reserved. No reuse allowed without permission.

we restricted our search to these three variants of the given architecture, and trained a handful of versions of each variant with different hyperparameter settings. To evaluate the generalization performance of the models, we divided our data at two levels; A reference dataset (Figure 1 and Supplementary Table 1) and an external dataset (Figure 2a and Supplementary Table 2). In the reference dataset, we evaluated the performance of the trained models on an independent test split from known scanners with an age distribution resembling the training split. We then tested model performance on the external dataset compiled from different sources, originating from scanners unseen by the models during training with a divergent age distribution.

\section{Superior generalization performance of SFCN-reg}

We used the training and validation sets to optimize and tune the models, and a conjunction of the test set and the external dataset in a final model comparison. For each model variant we trained three versions, with different hyperparameter settings, on the training data, and selected the best model based on the mean absolute error (MAE) on the validation set (Figure 1c and Supplementary table 8). We then compared the performances of the best version for each model variant on the test set. In this comparison the results mirrored those of the validation set, with SCFN-sm achieving the best result with an MAE of 2.23 years, followed by SCFN-reg with 2.47 years and SCFN-rank with 2.55 years (Figure 2b). Given the added complexity of including multiple datasets from a large range of scanners, we consider these results to be approximately on par with the MAE of 2.14 reported in the original SFCN paper ${ }^{21}$, and thus among the best performing models in the field ${ }^{34}$. Additionally, the heterogeneous origins of the dataset facilitate cross-site generalization, an essential property when training large multisite models ${ }^{35}$.

As a conclusive test of model generalization, we performed the same comparison on the external dataset, containing unseen data from different MRI scanners with an age distribution diverging from that of the reference dataset. SCFN-reg substantially outperformed the two alternatives, with an MAE of 3.90 compared to 5.04 and 5.82 for SFCN-sm and SFCN-rank respectively (Figure 2). While the performance of all models were lower on the external dataset, the extent of the generalization error was considerably different. When compared with MAEs from the test set, the average error of SFCN-reg increased by approximately half, while it more than doubled in SFCN-sm and SFCN-rank (Supplementary Table 9). This difference coincides with the architectural differences between the models: Where both the SFCN-sm and SFCN-rank used age-bins, with an output node for each age in its prediction range, SFCN-reg had a single output predicting a single continuous 
medRxiv preprint doi: https://doi.org/10.1101/2021.10.29.21265645; this version posted October 30, 2021. The copyright holder for this preprint (which was not certified by peer review) is the author/funder, who has granted medRxiv a license to display the preprint in

All rights reserved. No reuse allowed without permission.

number. Therefore, the predictions of the SFCN-reg reflect a simpler combination of the learned representations in the preceding layer of the model, which we hypothesize may be the reason for the improved generalization performance. In the subsequent applications we use the SFCN-reg version that achieved the best MAE on the external dataset. Additionally, to facilitate cross-study comparisons, we have compiled a range of performance metrics for our three models on the external dataset in Table 1.

\begin{tabular}{|c|c|c|c|c|c|c|}
\hline Model & MAE & RMSE & $\mathrm{R}$ & $\mathrm{R}^{2}$ & mRMSE & $\mathrm{RAE}$ \\
\hline SFCN-sm & 5.04 & 6.51 & 0.961 & 0.903 & 0.078 & 0.26 \\
\hline SFCN-reg & $\mathbf{3 . 9 0}$ & $\mathbf{5 . 1 1}$ & $\mathbf{0 . 9 7 5}$ & $\mathbf{0 . 9 4 0}$ & $\mathbf{0 . 0 6 1}$ & $\mathbf{0 . 2 0}$ \\
\hline SFCN-rank & 5.92 & 7.54 & 0.959 & 0.870 & 0.090 & 0.31 \\
\hline
\end{tabular}

Table 1: Predictive performance of the soft classifiation model (SFCN-sm), the regression model (SFCN-reg) and the ranking model (SFCN-rank) on the external dataset, originating from scanners which has not been seen by the models during training. Mean Absolute Error (MAE), Root Mean Squared Error (RMSE), R and R2 are sensitive to the age range of the dataset, while normalized RMSE (nRMSE) and Relative Absolute Error (RAE) are not, facilitating comparisons across datasets.

Observing that all the models performed worse in the external dataset than in the test set, we performed post-hoc analyses to further understand the causes underlying the generalization problems. Specifically, we tried isolating two sources of generalization error: Differences in population, represented here by age and sex distributions, and differences in scanners and acquisition protocols. We approached this by resampling two artificial datasets, both with participants previously unseen by the models. First, we sampled a dataset with an "Unknown population", with participants from the test set following the empirical age and sex distribution of the external dataset. Secondly, we created a dataset with "Unknown scanners", sampling participants from the external dataset while following the distributions of the test set (see Methods for further details). Due to the stratification used in the initial train/validation/test split the latter set also directly matches the distributions of the training set. For each of these two new datasets we computed an MAE per model, which naturally fell between the MAEs on the test set and on the external dataset. While this approach is exploratory and inherently limited to the characteristics and actual data points making up our datasets, the results clearly indicate that the main driver of generalization error is the unknown scanners (Table 2 and Supplementary Figure 1) which had higher errors (MAE $\left.\mathrm{US}_{\mathrm{S}}\right)$ than the unknown population $\left(\mathrm{MAE}_{\mathrm{UP}}\right)$ for all the models. Additionally, these two sources of generalization 
medRxiv preprint doi: https://doi.org/10.1101/2021.10.29.21265645; this version posted October 30, 2021. The copyright holder for this preprint (which was not certified by peer review) is the author/funder, who has granted medRxiv a license to display the preprint in perpetuity.

All rights reserved. No reuse allowed without permission.

\section{Test set}

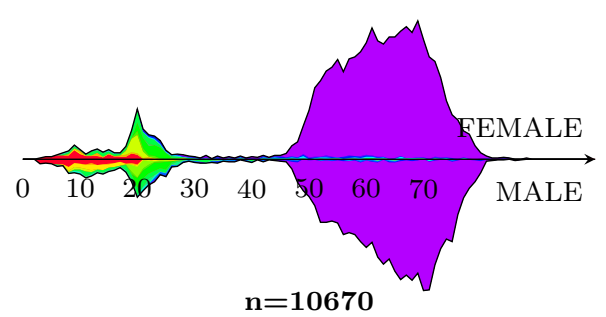

Colouring scheme is the same as used in Figure 1a
External dataset

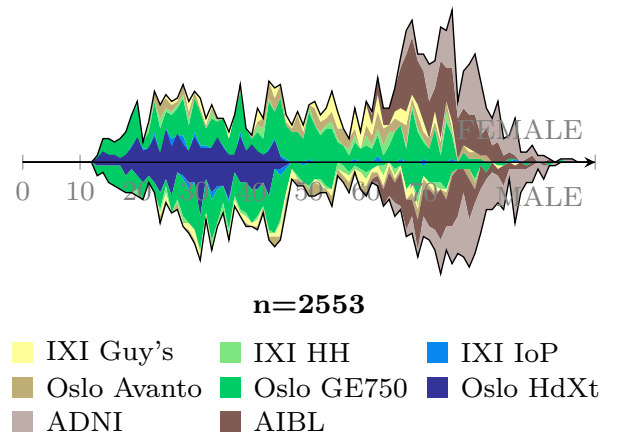

(a) Age distribution of the two datasets used in the comparison
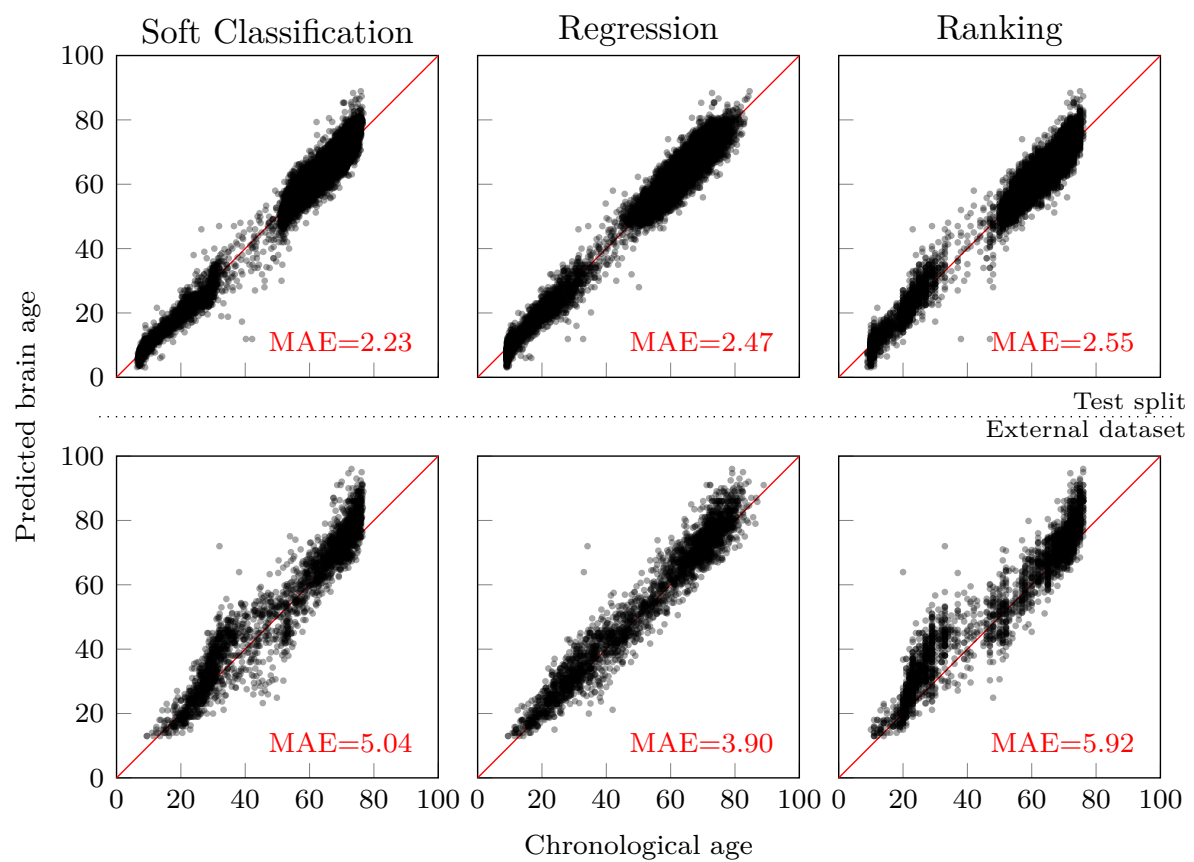

(b) Brain age predictions

Figure 2: The two datasets used in the model comparison, and the predictive performance of the three model variants. (a) For comparing the models we employed two distinct datasets: A test set sampled from the reference dataset, and an external dataset. The former was drawn using stratification, and as a consequence has an age distribution closely resembling the one seen in the full reference dataset. The latter was compiled from a different subset of datasets, and thus was acquired from different scanners. The age range of the external dataset is somewhat narrower, spanning a region of 13 to 95 years, and has a more uniform distribution. (b) The six scatter plots display the predictions of a given model on the x-axis, against the ground truth age of the participants on the y-axis. The top row contains the predictions of the three model variants on the test set, and the bottom row the external dataset. 
medRxiv preprint doi: https://doi.org/10.1101/2021.10.29.21265645; this version posted October 30, 2021. The copyright holder for this preprint (which was not certified by peer review) is the author/funder, who has granted medRxiv a license to display the preprint in

All rights reserved. No reuse allowed without permission.

error seem to work additively, with their sum closely matching the full generalization error observed in the external dataset. A final observation was that SFCN-reg handled both sources of error best, which is further evidence of its superior ability to generalize.

\begin{tabular}{|c|c|c|c|c|}
\hline Model & MAE $_{\text {TEST }}$ & MAE $_{U P}$ & MAE $_{\text {US }}$ & MAE $_{\text {EXTERNAL }}$ \\
\hline SFCN-sm & $\mathbf{2 . 2 3}$ & 3.48 & 3.84 & 5.04 \\
\hline SFCN-reg & 2.47 & $\mathbf{2 . 8 9}$ & $\mathbf{3 . 4 2}$ & $\mathbf{3 . 9 0}$ \\
\hline SFCN-rank & 2.55 & 3.95 & 4.32 & 5.92 \\
\hline
\end{tabular}

Table 2: Mean Absolute Errors measured for the three model variants on the test set, drawn from the same distributions of scanners and ages as the training dataset, the "Unknown population" dataset (MAE $\mathrm{EP}_{\mathrm{P}}$ ) and the "Unknown scanners" dataset $\left(\mathrm{MAE}_{\mathrm{US}}\right)$, both representing a single source of generalization error, and the external dataset, different from the training set in both regards.

\section{Brain age predictions associate with biological phenotypes and lifestyle factors}

Next, we validated the relevance of the model predictions by correlating their deviations from chronological age with an array of phenotypes in a phenome-wide association study. We performed this analysis in the subset of the UKBB data that was not used for brain age modelling or validation $(n=8066)$, and tested associations with all the biological phenotypes and lifestyle variables accessible, manually divided into thirteen thematic categories for interpretability (Supplementary Table 7). For each phenotype we computed a univariate correlation while correcting for age and $\operatorname{sex}^{36,37}$, and assessed its significance using a Bonferroni-corrected p-value threshold of $p<1.26 \times 10^{-4}$ (see Methods). All continuous variables were standardized, such that their effect sizes denote the impact a one standard deviation increase has on the brain age delta. In general, our results corroborated several findings derived from previous studies using smaller samples (Figure 3a). We observed significantly higher delta in participants with high blood pressure $\left(\beta=0.41, p=1.86 \times 10^{-7}\right)$, those currently on blood pressure medication $\left(\beta=0.54, p=1.17 \times 10^{-10}\right)$, and a positive correlation with blood pressure readings (diastolic $(\mathrm{DBP}): \beta=0.15, p=2.53 \times 10^{-6}$, systolic $\left.(\mathrm{SBP}): \beta=0.16, p=3.30 \times 10^{-6}\right)$. The associations with the largest effects indicated higher delta in patients with a diabetes diagnosis $\left(\beta=0.74, p=2.25 \times 10^{-7}\right)$ or diabetes-related eye problems $\left(\beta=1.78, p=4.59 \times 10^{-7}\right)$. Among the biochemical measurements, significant associations with brain age delta were found for 
blood glucose levels $\left(\beta=0.23, p=3.18 \times 10^{-11}\right)$, Insulin-Like Growth Factor-1 levels $(\beta=-0.22$, $\left.p=5.81 \times 10^{-11}\right)$, glycated haemoglobin levels $\left(\beta=0.16, p=7.97 \times 10^{-7}\right)$ and mean corpuscular volume $\left(\beta=0.13, p=3.33 \times 10^{-5}\right)$. Associations with variables we categorized as related to diet and lifestyle were dominated by previous smoking, with a positive correlation with number of cigarettes per day (absolute pack years: $\beta=0.24, p=8.55 \times 10^{-5}$, pack years as proportion of age: $\beta=0.26$, $\left.p=2.41 \times 10^{-5}\right)$ and age stopped smoking $\left(\beta=0.25, p=7.59 \times 10^{-5}\right)$. We also observed significant associations with average weekly beer and cider intake $\left(\beta=0.21, p=1.13 \times 10^{-7}\right)$ and alcohol intake frequency $\left(\beta=0.12, p=1.09 \times 10^{-4}\right)$, cereal intake $\left(\beta=-0.16, p=7.61 \times 10^{-7}\right)$ and participation in "Other group activity" (e.g. social activities not related to a sports or social club, religious group or adult education, $\left.\beta=-0.27, p=5.91 \times 10^{-5}\right)$. Further, we observed a significant correlation with the number of people living in the participants household $\left(\beta=-0.13, p=8.20 \times 10^{-5}\right)$ and higher deltas in those born outside the United Kingdom and the Republic of Ireland (compared to the baseline group born in England, $\left.\beta=0.62, p=4.62 \times 10^{-6}\right)$. An overview of all the 394 associations can be found in Supplementary Table 12.

\section{Transferring brain age predictions to developmental and degenerative brain disorders}

For six different disorders we compiled a patient cohort and a matched control group, and calculated a brain age delta per participant based on the prediction from SFCN-reg (Supplementary Figure 4). In all control groups, the brain age prediction accuracy was approximately the same as for the full test set (MAEs=2.91-4.05, Supplementary Figure 3). Patients with MS showed significantly higher brain age estimates than their matched healthy controls (brain age group mean difference $\Delta=4.42$ years, $p=1.71 \times 10^{-22}$, Cohen's $\left.d=0.87\right)$. A similar pattern was also observed for patients with $\operatorname{AD}\left(\Delta=2.81, p=4.20 \times 10^{-20}, d=0.59\right)$, MCI $\left(\Delta=2.13, p=1.25 \times 10^{-15}, d=0.46\right)$ and $\mathrm{SCZ}\left(\Delta=1.40, p=4.29 \times 10^{-5}, d=0.34\right)$. For the individuals with mood disorders (MOOD, see Methods) this difference was the smallest $(\Delta=0.64, p=0.04, d=0.17)$, while the difference was not significant for patients with a mix of psychotic diagnoses (PSY) $(\Delta=0.74, p=0.15$, $d=0.20)$. Both the relative ordering of the disorders in terms of group difference, the magnitude of the disparities, and the observed significance resemble a previous study ${ }^{8}$ using a different model based on a smaller dataset.

To demonstrate the predictive power of our best performing pretrained brain age model for clinical conditions, we trained multiple binary classifiers to predict whether a participant had a 
medRxiv preprint doi: https://doi.org/10.1101/2021.10.29.21265645; this version posted October 30, 2021. The copyright holder for this preprint (which was not certified by peer review) is the author/funder, who has granted medRxiv a license to display the preprint in perpetuity.

All rights reserved. No reuse allowed without permission.

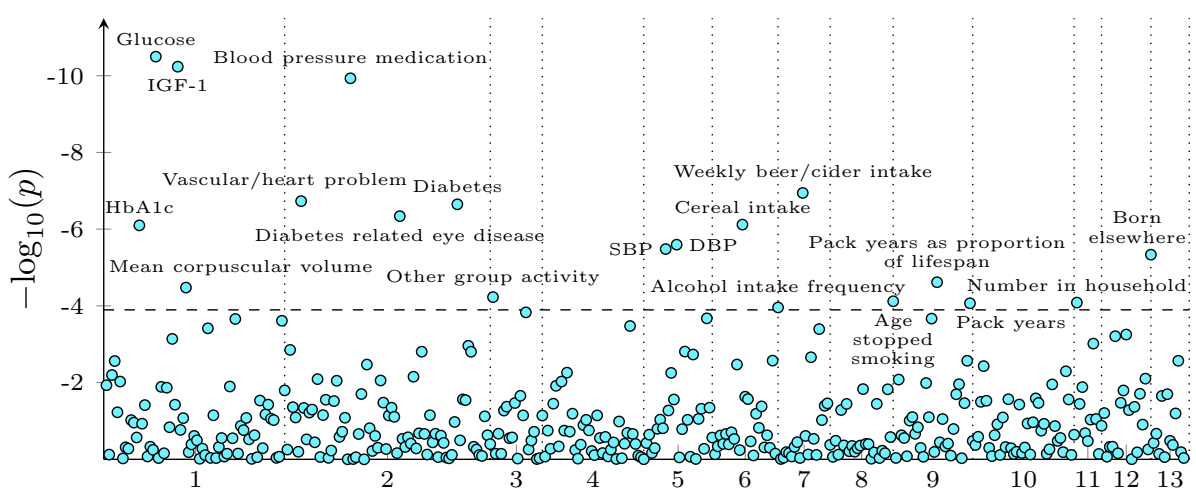

(a) Significances of the PheWAS correlations

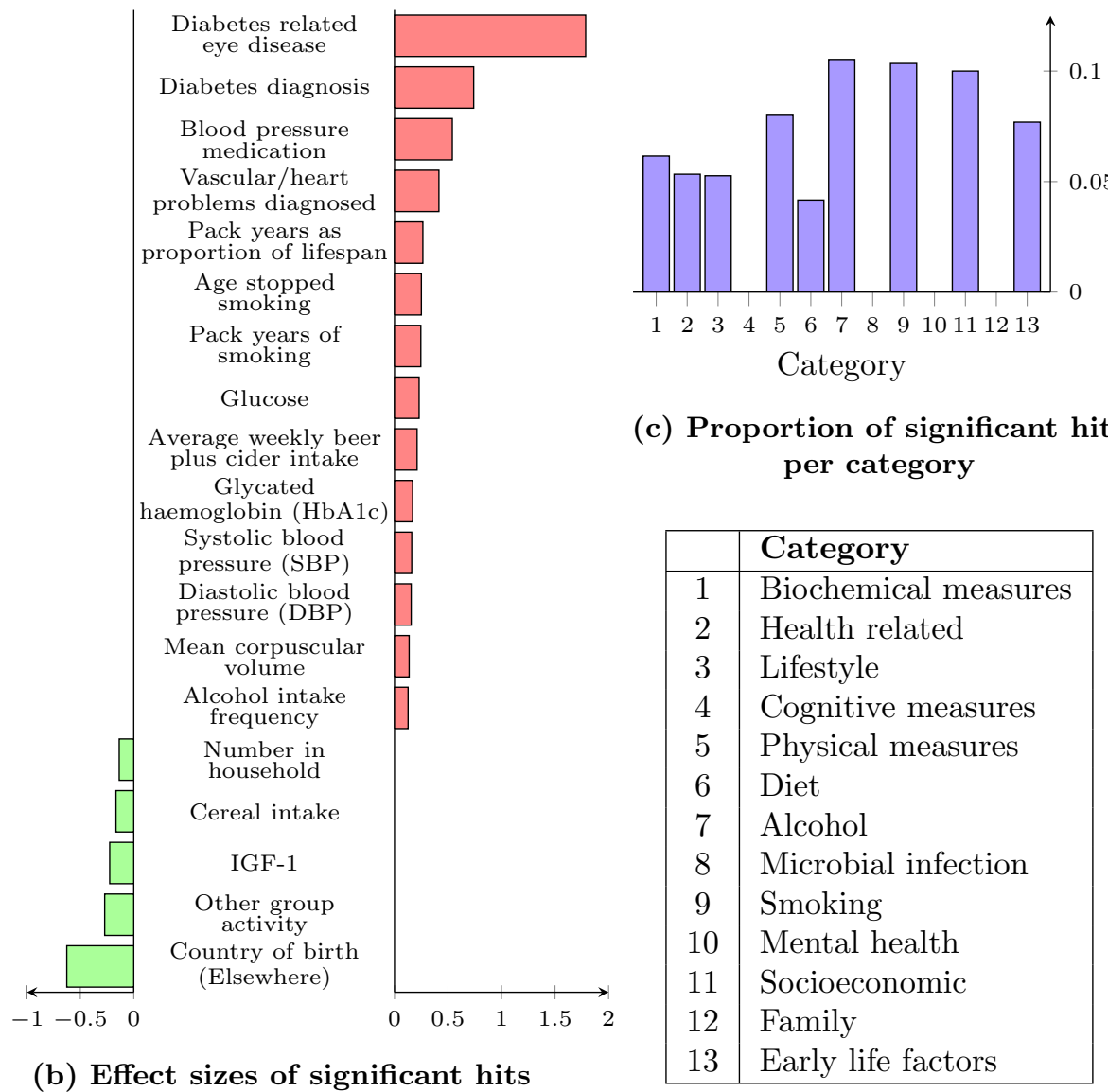

Figure 3: Associations between the brain age delta and a wide range of phenotypes. We correlated the brain age delta originating from the SFCN-reg with 394 phenotypic variables in the test set portion of the UKBB, categorized into thirteen categories. (a) A Manhattan plot visualizing the significances of the 394 associations. Using a Bonferroni-corrected threshold, 19 of these were found to be significantly associated with the delta. (b) The effect sizes of the significant associations. For binary variables the effect size expresses the mean difference between the groups, while for continuous variables it denotes the change in brain age delta associated with a one standard deviation increase. (c) For each of the thirteen categories we calculated the proportion of significant hits by dividing the number of significant hits within that category with the total number of variables in the same category. 
medRxiv preprint doi: https://doi.org/10.1101/2021.10.29.21265645; this version posted October 30, 2021. The copyright holder for this preprint (which was not certified by peer review) is the author/funder, who has granted medRxiv a license to display the preprint in

All rights reserved. No reuse allowed without permission.

diagnosis or not (Figure 1c, Figure 4 and Methods). We used logistic regression models with an $l_{1}$-penalization for this purpose (LASSO models), optimized via a nested cross validation procedure (Supplementary Figure 5), and started with a baseline model classifying participants based only on age and sex. The second model included the brain age delta originating from the brain age prediction of SFCN-reg, and the third model replaced the brain age delta by 64 features encoded in the second to last layer of the same model (Figure 4b and Methods). Across the six disorders, the baseline models achieved an area under the receiver operating curve (AUCs) ranging from 0.47 to 0.54 , indicating that our matching procedure was satisfactory. Using the second set of models, quantifying the predictive power of the brain age deltas in Supplementary Figure 4, greatly improved the prediction performance when compared to the corresponding baseline models for MS (AUC=0.71 vs. 0.50$)$, $\mathrm{AD}(0.69$ vs. 0.51$)$ and MCI (0.65 vs. 0.54$)$; but to a minimal extent for SCZ (0.58 vs. 0.50), MOOD (0.55 vs. 0.52), and PSY (0.52 vs. 0.47). A third set of classifiers were implemented in a strict transfer learning context, utilizing the 64 features of the second-to-last layer of SFCN-reg as predictors (Methods). These features represent high-level, data-driven abstractions of the brain imaging data, and underlie the singular brain age prediction. We refer to this variant of transfer learning as strict because we kept the weights of the initial brain age model locked while optimizing for the new binary objective, which in turn allow us to keep treating these as ageing features and thus promote interpretability. While this complicates contextualizing the performance of our models in terms of existing case-control classifiers, it gives us an indication of the information content of these learned features. This third set of models improved AD prediction substantially (AUC=0.83), and also were notably better for MS (0.79), MCI (0.73) and PSY (0.62), while only a marginal improvement was observed in SCZ (0.62) and MOOD (0.59). Overall, our results show that our brain age model can be transferred to make case-control predictions of these common clinical brain disorders.

\section{Discussion}

Brain maturation and ageing, and its interactions with clinical brain disorders and conditions, are complex processes with pivotal environmental and genetic contributions ${ }^{38,39}$. Brain age prediction and the accompanying brain age delta has the potential to provide intuitive and useful measures for summarizing individual brain aberrations. However, technical differences between studies, e.g. the use of different scanners and MRI scan parameters, have represented challenges for the direct generalization and applicability of brain age models based on large training sets. We assembled 
medRxiv preprint doi: https://doi.org/10.1101/2021.10.29.21265645; this version posted October 30, 2021. The copyright holder for this preprint (which was not certified by peer review) is the author/funder, who has granted medRxiv a license to display the preprint in

perpetuity.

All rights reserved. No reuse allowed without permission.

MS

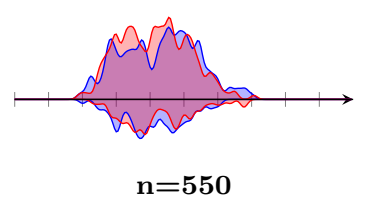

MCI

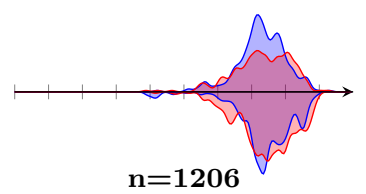

MOOD

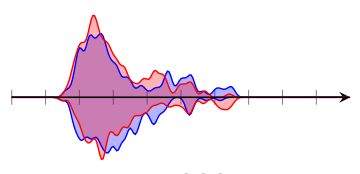

$\mathbf{n}=\mathbf{3 3 8}$
$\mathrm{AD}$

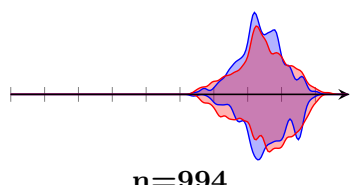

$\mathrm{SCZ}$

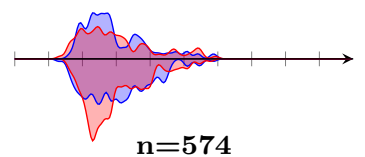

PSY

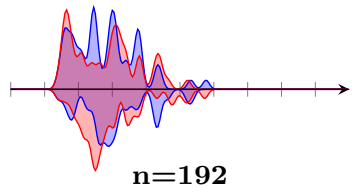

$\mathrm{n}=192$

(a) Patient and control group age distributions
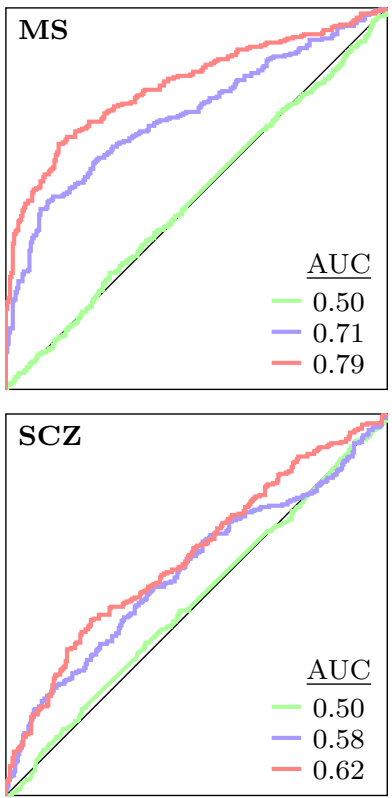
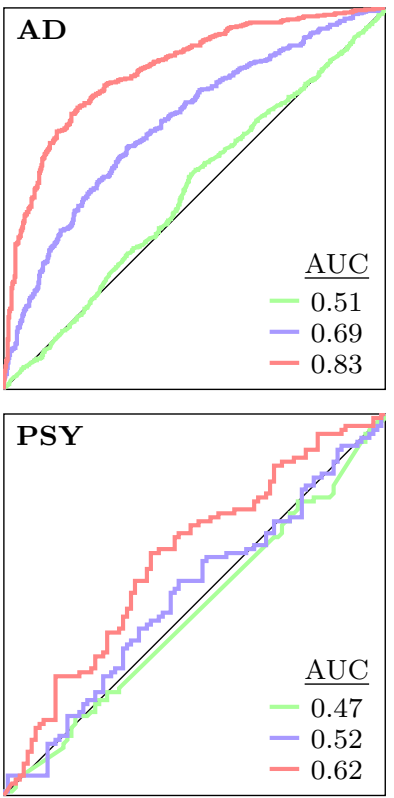

Age<smiles></smiles>

- Baseline model

Brain age model

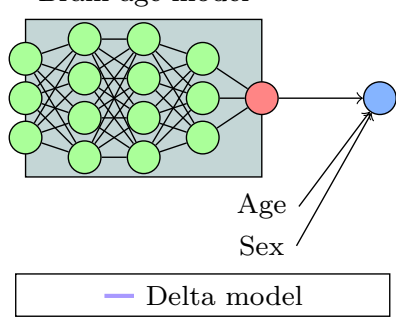

Brain age model

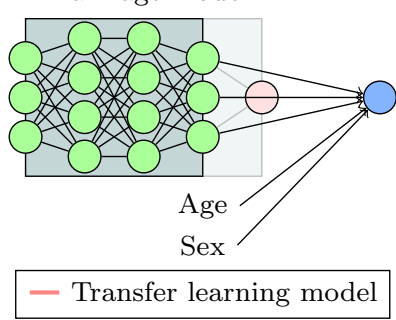

(b) Conceptual model compositions
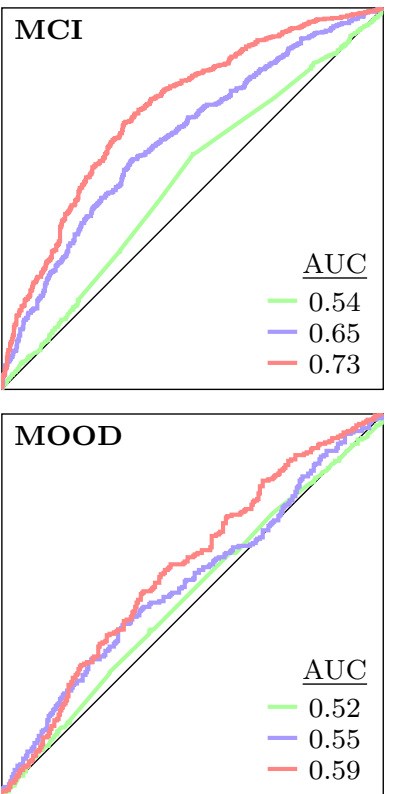

(c) ROC curves for the predictors

Figure 4: The datasets and models used for clinical predictions, and an overview over their performance. We applied the best performing SFCN-reg in a clinical setting by training binary classifiers to discriminate between patients and controls for multiple common brain disorders, using different levels of information from the brain age model. (a) To avoid biases we used a strict matching procedure, drawing a set of controls for each scanner-specific patient dataset matching its empirical age and sex distribution. (b) We trained three logistic regression models for each disorder to compare their performance. The first used age and sex as predictors in a baseline model, the second included the brain age delta, quantifying its value as a clinical predictor, and the third used internal features of the brain age model in a transfer learning setting. (c) For each disorder we compared the binary models using AUCs, indicating the information content of the predictors in relation to the given disorder. 
medRxiv preprint doi: https://doi.org/10.1101/2021.10.29.21265645; this version posted October 30, 2021. The copyright holder for this

preprint (which was not certified by peer review) is the author/funder, who has granted medRxiv a license to display the preprint in

All rights reserved. No reuse allowed without permission.

a large and diverse neuroimaging dataset to train multiple state-of-the-art deep learning models for brain age prediction, and extensively tested their ability to generalize, and their sensitivity to various common brain disorders. Our best model, the SFCN-reg, showed superior performance on an external dataset with differing scanners and age distributions compared to that of the training set, enabling it for applications in other datasets. We then demonstrated the relevance of the model predictions by showing associations between the brain age delta and a range of complex human traits and health outcomes in a population sample. Lastly, we transferred the trained SFCN-reg to clinical data in a transfer learning setting, showing that both the brain age delta and the internal features learned by the model have predictive value when differentiating between controls and patients with common brain disorders.

In our experiment the SFCN-reg outperformed the other models in terms of generalization performance. The age prediction accuracy on the test data $(\mathrm{MAE}=2.47)$ is among the best in the field ${ }^{34}$. Crucially, the prediction accuracy on data coming from unknown scanners (MAE $=3.90)$ fares very favourably when compared to other studies attempting to transfer between datasets of similar size and complexity $30,32,34,40,41$. Using data from different scanners, protocols and populations in neuroimaging comes with the problem of modelling the effects of these appropriately ${ }^{18,42}$. Previous brain age studies have often explicitly included a scanner term in the modelling or corrected the computed brain age for various biases ${ }^{43}$. Recent approaches have tried to address this problem directly via specific deep learning paradigms ${ }^{44}$. Our results show that given sufficiently large and heterogeneous training data, deep CNNs achieve state-of-the-art performance for brain age predictions even when scanner effects are not explicitly modelled, and more importantly that this performance translates to scanners and protocols that are unknown to the model. This suggests that the representations learned by the model are dominated by age-related variance, not scanner-dependent artefacts, an extension of the model robustness shown in earlier studies ${ }^{19}$. From a practical point of view this suggests that our trained model may be employed in other applications in new datasets, without the need for retraining or applying corrective procedures.

Evaluating the biological relevance of the brain age predictions is essential to further understand and trust these models. Therefore, we correlated brain age delta obtained from the SFCN-reg with a wide range of phenotypes in a subset of the UKBB not used for model training. When applying a Bonferroni-corrected threshold, we found nineteen significant hits spread across seven of our thirteen categories. Almost all of these have in earlier studies been found to have a relationship with ageing, either generally or specifically in the brain, or with brain health and/or cognitive function: Glucose level ${ }^{45}$, Insulin-like Growth factor- $1^{46}$, and glycated haemoglobin ${ }^{47}$ are known to 
change with age, and corpuscular volume has been associated with cognitive functioning ${ }^{48}$. Lifestyle factors involving alcohol and smoking impact various biological and bodily ages ${ }^{45}$, including that of the brain ${ }^{49-51}$. Elevated blood pressure and other cardiovascular risk factors have established associations with increased brain age $\mathrm{s}^{50,52}$, and an increase in predicted age has been observed in patients with diabetes ${ }^{53}$. In sum, this analysis shows that our model makes biologically meaningful predictions. Further, transferring the model to unseen datasets comprised of patients with different clinical conditions and matched healthy peers revealed both high accuracy in terms of age prediction and higher deltas among patients with brain disorders, in line with previous studies ${ }^{8}$. Importantly, the data in the case-control datasets were obtained from scanners not included in the training set, supporting that the model generalizes to previously unseen scanners; a highly valuable asset.

Compared to the all-in-one brain age delta, a single number describing the difference between apparent and chronological age, our results showed increased predictive value for MS, AD and MCI when using the internal representations of the SFCN-reg underlying the brain age predictions. This supports the view that for some applications the constituent components of the singular brain age delta are relevant beyond the age prediction alone ${ }^{54}$. The innate ability of deep neural networks to form abstractions of the brain at different spatial resolutions throughout the layers of the model may help disentangle individual differences in neurodevelopmental and age-related processes related to complex disorders and traits. Furthermore, we see this result as evidence that deep learning models trained to predict age in large multisite datasets constitute excellent starting points for transfer learning, which can subsequently be fine-tuned to a variety of tasks.

There is an ongoing discussion in the field on whether brain age models that are precise, or those that allow for sufficient variance in their single-subject predictions, are the most useful in a downstream analysis of behavioural and clinical traits ${ }^{32,55,56}$. An argument for a model which allows for more variation (a looser fit) is that this would more accurately depict brain age as a complex process which appears differently in different individuals. One challenge with this approach is that the brain age delta is a residual, and recognizing what portion of this error comes from biological variation and what is modelling imprecision is practically unfeasible. As more complex models such as deep CNNs become competitive for brain age modelling, it becomes possible to minimize the overall model error, including the methodological portion, while still allowing the model to accurately represent the necessary biological variability internally.

There are some limitations of the present study which we acknowledge here. Given the computational cost of training complex deep learning models on such a large dataset we restrained our study to a limited number of possible models, both in terms of model architectures and hyperparameter 
medRxiv preprint doi: https://doi.org/10.1101/2021.10.29.21265645; this version posted October 30, 2021. The copyright holder for this

preprint (which was not certified by peer review) is the author/funder, who has granted medRxiv a license to display the preprint in

All rights reserved. No reuse allowed without permission.

settings. With further increases in sample sizes and diversity, deeper architectures may be sensible, as the risk of overfitting is directly alleviated by the larger datasets. While we refer to our dataset as diverse, most participants included in the study are of white European background. However, this limitation is not specific to the present study, and global collaborations are needed to build models that generalize across cultural and genetic backgrounds ${ }^{57}$. Relatedly, the current investigation included T1-weighted MRI data only. Future integration of information spanning various imaging modalities may increase both age prediction accuracy and the sensitivity and specificity of various biological and clinical traits and conditions ${ }^{11,52,54,58}$. To evaluate the clinical relevance of the brain age model in the context of disorders we employed transfer learning in a strict way, by training LASSO models on top of the SFCN architecture. This setup increases the interpretability of the transfer process as the lower-level representations of our model used as predictors capture age-related features of the brain. In turn, this allows for the interpretation that illnesses that are predictable by the model must also rely on these representations, and thus implicitly relate to age. There are multiple steps which could have been taken instead to maximize predictability, a natural starting point is to fine-tune the entire model ${ }^{32}$. Lastly, in order to reach broad adoption of these models and, ultimately, approach clinical usability, a better understanding of the regional patterns driving the prediction, their specific biological significance and how it changes across time and contexts is needed $^{59}$.

In conclusion, we have trained multiple variants of a deep neural network to predict brain age on a large and heterogeneous sample of raw structural MRI data, and observed distinct differences in their ability to generalize to unseen samples and scanners. The predictions of our best model were linked to biochemical biomarkers, cardiovascular risk factors, smoking and alcohol intake, among others. Using transfer learning, we demonstrated that clinical conditions with a neurodevelopmental or neurodegenerative aetiology were predictable by our model, initially trained to predict age. Jointly, these findings add to the growing literature documenting the tremendous potential of advanced techniques for statistical learning to decode biologically and clinically relevant information from brain MRI scans. 


\section{Methods}

\section{Data}

All data sets used in the present study have been obtained from previously published studies which have been approved by their respective institutional review board or relevant research ethics committee.

\section{Reference dataset}

The reference dataset used for training the brain age models was T1-weighted MRI scans derived from 21 non-overlapping and publicly available datasets (total $n=53542$; female $\mathrm{n}=27715$ ) of healthy individuals, with ages ranging from 3 to 95 years (Figure 1 and Supplementary Table 1). The age distribution can be seen in Figure 1a. The younger age-range (3-30 years) was mainly composed of participants from multiple different datasets. Though the older age-range (40-80) also included multiple datasets, UKBB accounted for most of these participants. The most sparsely populated age-ranges were in the very young (147 participants with age $\leq 5)$, very old (17 participants with age $\geq 85$ ), and in midlife (42 participants with $35 \leq$ age $<45$ ). For each of the datasets, participants that had one or more psychiatric, neurological and/or other relevant diagnoses (Supplementary Table 4), and those withdrawn from the respective study were excluded before model training. In addition, for participants having multiple brain scans, the baseline data were used, such that in the final dataset each data point represents a unique participant drawn from a normative population.

\section{External dataset}

To evaluate the generalizability of our trained brain age prediction model, an external dataset was collected (Supplementary Table 2). This dataset included the IXI project (Supplementary Table 5) and healthy controls from the datasets underlying the clinical data described below (total $\mathrm{n}=2553$ ). Importantly, the external dataset contained images generated by scanners not used in the reference dataset, and subsequently unknown to the models during training and validation. This dataset can be seen as having a more uniform age distribution (Figure 2a), meaning that our test would capture whether any given model relies too heavily on information observed in the training data.

\section{Clinical data}

The clinical data consisted of six patient cohorts diagnosed with MCI, AD, MS, SCZ, a mix of psychotic diagnoses and mood disorders, where the latter was a combination of two cohorts with 
medRxiv preprint doi: https://doi.org/10.1101/2021.10.29.21265645; this version posted October 30, 2021. The copyright holder for this preprint (which was not certified by peer review) is the author/funder, who has granted medRxiv a license to display the preprint in

All rights reserved. No reuse allowed without permission.

depression and bipolar disorder (Figure 2a and Supplementary Table 3). The individual cohorts were compiled from ADNI, AIBL, and multiple scanners at the Oslo University Hospital (Supplementary Table 5 and 6 ). In addition to the patients, we used healthy controls from the same scanners in the external dataset to create matched control groups for the clinical predictions.

\section{Quality control}

To ensure data quality, we executed a quality control (QC) pipeline, consisting of checking whether any of the image preprocessing steps failed, and a manual control via visual inspection. To take advantage of as much data as possible this manual control was lenient, removing samples where either a significant portion of the brain was missing, or where the orientation of the head was dramatically off, and resulted in dropping only 39/53581 participants in the reference dataset (0.07\%) and 1/2554 participants in the external dataset $(0.03 \%)$.

\section{Image preprocessing}

We first performed skull-stripping with the FreeSurfer 5.3 auto-recon pipeline ${ }^{60}$ to produce a brainmask, minimizing the amount of non-brain information in the data, then reoriented the images to the standard $\mathrm{FSL}^{61}$ orientation using fslreorient2std. The resulting images were linearly registered to the MNI152 space using FLIRT ${ }^{62}$ with linear interpolation and the default $1 \mathrm{~mm}$ FSL template (version 6.0). We cropped away borders of [6:173, 2:214, 0:160] voxels, in the sagittal, coronal and axial dimensions respectively. This cropping yielded the smallest cuboid with marginal loss of brain-related information across the dataset, minimizing the memory footprint of the models during training. As a last preprocessing step the voxel intensity values of all brain images were normalized to the range $[0,1]$.

\section{Brain age models}

The state-of-the-art network architecture, the Simple Fully Convolutional Network (SFCN) ${ }^{21}$, was implemented as the backbone in all our brain age models. The SFCN architecture consists of a $\mathrm{VGG}^{63}$-like structure, with five repeated convolutional blocks, each with a three-dimensional convolutional layer with a filter size of $(3,3,3)$, a batch normalization layer, rectified linear activation function (ReLU) activation, and a max-pooling layer with a pooling size of $(2,2,2)$ (Figure 1b). The model then has a channel-wise convolutional layer, a last batch normalization layer and a global average pooling layer. From this backbone we defined three end-to-end variants for brain 
age prediction: The original soft classification model (SFCN-sm), a regression variant with a single output node with a linear activation (SFCN-reg) and a ranking model from the general age-regression literature (SFCN-rank) ${ }^{33}$, an approach which has also been successful for brain age predictions ${ }^{64}$. All the model definitions rely on a matrix $X$ of dimensions $[N, h, w, d]$ containing MRI images as input, an N-dimensional vector age $=\left[\operatorname{age}_{0}\right.$, age $_{1}, \ldots$, age $\left._{N-1}\right]$ containing the ground truth ages of the participants to compute its loss, and are ultimately able to produce an $\mathrm{N}$-dimensional vector age $=\left[a \mathrm{ag}_{0}, \mathrm{age}_{1}, \ldots, \mathrm{age}_{N-1}\right]$ with a single brain age prediction per participant (although this is not necessarily the direct output of the model).

\section{SFCN-sm}

The soft classification variant formulates the age regression problem as a multiclass classification problem, by having $m=\lceil\max ($ age $)\rceil-\lfloor\min ($ age $)\rfloor$ output neurons, where $\lceil\cdot\rceil$ and $\lfloor\cdot\rfloor$ denote the ceiling and floor operators respectively. It is denoted as soft because it uses a target vector per participant generated by a normal distribution centered around the ground truth age, instead of the one-hot encoding used in regular classification

$$
y_{i}=\mathcal{N}\left(\operatorname{age}_{i}, 1\right)
$$

The predictions of the model are similarly a vector of length $m$ with a softmax activation

$$
\hat{y}_{i}=\left[\hat{y}_{i, 0}, \hat{y}_{i, 1}, \ldots, \hat{y}_{i,(m-1)}\right], \sum_{j=0}^{m-1} \hat{y}_{i, j}=1 .
$$

The loss for a single datapoint is the Kullback-Leibler divergence between the two vectors

$$
\operatorname{loss}_{i}=\mathrm{KL}\left(y_{i} \| \hat{y}_{i}\right)
$$

The final age-prediction of a participant is calculated as a weighted sum of the prediction vector

$$
a \hat{g} e_{i}=\lfloor\min (\text { age })\rfloor+\sum_{j=0}^{m-1} j \hat{y}_{i, j} .
$$

\section{SFCN-reg}

The regression variant has a single output neuron predicting a single value $\hat{y}_{i}$ per participant, limited to the range $(\min (\operatorname{age}), \max ($ age $))$ with a bounded ReLU activation. The value of $\hat{y}_{i}$ can be used 
directly as the predicted age for a participant, $a \hat{g} e_{i}=\hat{y}_{i}$. During training, the model optimizes the mean squared error.

\section{SFCN-rank}

The ranking model formulates the age regression problem as a set of binary "Is participant $X$ older than age $y$ ? "-questions. Like the soft classification model, the model has $m=\lceil\max ($ age $)\rceil-$ $\lfloor\min ($ age $)\rfloor$ output neurons, each representing one such binary question. The target vector for a participant is a binary vector of length $m$, with a 1 in bins corresponding to ages younger than the participants age and 0 in the rest.

$$
y_{i}=\left[y_{i, 0}, y_{i, 1}, \ldots, y_{i,(m-1)}\right], y_{i, x}=\left\{\begin{array}{rr}
1 & \lfloor\min (\text { age })\rfloor+x \leq \text { age }_{i} \\
0 &
\end{array}\right\}
$$

For each participant the model predicts a vector of length $\mathrm{m}$, where each output neuron is limited to the range $[0,1]$ by a sigmoid activation

$$
\hat{y}_{i}=\left[\hat{y}_{i, 0}, \hat{y}_{i, 1}, \ldots, \hat{y}_{i,(m-1)}\right], \hat{y}_{i, x} \in[0,1] .
$$

The model optimizes the mean binary cross entropy across all the output neurons

$$
\operatorname{loss}_{i}=-\frac{1}{m} \sum_{j=0}^{m} y_{i, j} \log \left(\hat{y}_{i, j}\right)+\left(1-y_{i, j}\right) \log \left(1-\hat{y}_{i, j}\right)
$$

To calculate a predicted age for the model we sum up the number of age bins for which the model predicts that a participant is older than the given age (it is worth noting that this limits the model to integer predictions)

$$
a \hat{g} e_{i}=\lfloor\min (\text { age })\rfloor+\sum_{j=0}^{m} \mathbb{1}_{x \geq 0.5}\left(\hat{y}_{i, j}\right)
$$

\section{Brain age model training and comparison}

All brain age models were trained on 2 NVIDIA V100 GPUs with 32GB memory, using the Keras ${ }^{65}$ interface of Tensorflow $2.3^{66}$ on top of cuda 10.0. Using a batch size of 14 the models took approximately 1 second per step, translating into roughly 45 minutes per epoch or about 2.5 days per full training session. To train the brain age models, $80 \%(n=42829)$ of the reference dataset was used for model building (training and validation) and $20 \%(\mathrm{n}=10713)$ for testing. Among the data for model 
medRxiv preprint doi: https://doi.org/10.1101/2021.10.29.21265645; this version posted October 30, 2021. The copyright holder for this preprint (which was not certified by peer review) is the author/funder, who has granted medRxiv a license to display the preprint in

All rights reserved. No reuse allowed without permission.

building $80 \%(\mathrm{n}=34285)$ and $20 \%(\mathrm{n}=8544)$ were used for training and validation of the models, respectively (Figure 1c and Supplementary Table 1). Before these splits, the data was stratified by age and original study to ensure that all subsets had resembling age distributions and came from multiple scanners. Given the great computational cost of model training, determining optimal hyperparameter values by searching over the full configuration space for each model is impractical. Instead, we employed post-hoc heuristics, i.e., tweaking the models based on previous runs. For each training run we trained the model from scratch (with randomly initialized parameters) for 80 epochs, optimized by vanilla stochastic gradient descent, employing an annealing, step-wise, learning rate schedule. This schedule had three steps, reducing the learning rate by a factor of 3 after epochs 20 , 40 and 60 . The initial learning rate was found independently for each model variant using a learning rate sweep ${ }^{67}$ (Supplementary Figure 7). We used mean absolute error (MAE) on the validation split to determine the best epoch for each run. We also report RMSE, R, $\mathrm{R}^{2}$ for all models in Table 1, and to enable comparisons with other studies with possibly different age ranges the normalized measures normalized RMSE (nRMSE)

$$
\text { nRMSE }=\frac{\text { RMSE }}{\max (\text { age })-\min (\text { age })}
$$

and Relative Absolute Error (RAE)

$$
\mathrm{RAE}=\frac{\sum_{i=0}^{N}\left|\hat{y}_{i}-y_{i}\right|}{\sum_{i=0}^{N}\left|y_{i}-\bar{y}\right|}
$$

The first model we trained was SFCN-sm with the hyperparameters specified in the original SFCN paper $^{21}$. Seeing that this model was underfitting we relaxed the regularization for a second run of the same model, and subsequently a third. The two hyperparameters we tuned in this process were the weight decay, and the dropout rate between the two final layers of the model. Having trained three soft classification models, we moved on to train three regression models and three ranking models using these same heuristics (Supplementary Table 8). To select a candidate model for each variant we compared the MAEs on the validation split. In the final model selection, we compared the MAEs of the candidate models for each variant on the test set and the external dataset. 
medRxiv preprint doi: https://doi.org/10.1101/2021.10.29.21265645; this version posted October 30, 2021. The copyright holder for this preprint (which was not certified by peer review) is the author/funder, who has granted medRxiv a license to display the preprint in

All rights reserved. No reuse allowed without permission.

\section{Post-hoc generalization analysis}

To study the causes of the differences in generalization, we designed an experiment to isolate the underlying sources of this error. Based on previous knowledge of the problems of new scanners, and the predictions of the models at different ages (Figure 3) we specifically targeted two possible sources: Differences in population, represented by different distributions of age and sex, and data coming from unknown scanners. For each source we sampled an artificial, bootstrapped dataset based on our existing data. For the "Unknown population" dataset we sampled participants from the test set (originating from the reference dataset), to match the empirical age and sex distribution of the external dataset. Similarly, for the "Unknown scanners" dataset we sampled participants from the external dataset (coming from scanners unknown to the model) to match the age and sex distribution of the test set (and thus also the training set). The idea behind both datasets is to isolate a single source of generalization error. For robustness, we bootstrapped each of these two artificial datasets 100 times and reported the mean MAE achieved by the different models. Each sample was drawn probabilistically, with replacement, with the probability of drawing participant $x$ of age $x_{a}$ and sex $x_{s}$ from dataset source based on the age and sex distribution of dataset target given by

$$
p(x)=\frac{\hat{P}_{\text {target }}\left(x_{a}, x_{s}\right)}{\hat{P}_{\text {source }}\left(x_{a}, x_{s}\right)}
$$

where $\hat{P}_{\text {dataset }}\left(x_{a}, x_{s}\right)$ denotes the proportion of participants in dataset dataset with age $x_{a}$ and sex $x_{s}$. In "Unknown population", the test set plays the role of source and the external dataset is the target, while this is switched for "Unknown scanners".

\section{Phenome-wide association study}

In the PheWAS we calculated the univariate correlation between the brain age delta and 402 phenotypic variables from the UKBB, manually divided into thirteen thematically defined categories for interpretability (Supplementary Table 7). We performed this analysis in the UKBB portion of the test split $(\mathrm{n}=8066)$, and used all the variables available to us at the time. We encoded all phenotypic variables according to the PHESANT ${ }^{68}$ datatypes, and removed non-informative levels (Supplementary Table 10) based on the UKBB coding schemes. Additionally, we re-coded the ordinal variables as categorical or continuous by hand (Supplementary Table 11). Variables which were impossible to model (i.e. singular or all missing values) were discarded. We then fitted a linear model per variable, modelling the delta as a function of the given covariate, age and sex, using the Python statsmodels 
medRxiv preprint doi: https://doi.org/10.1101/2021.10.29.21265645; this version posted October 30, 2021. The copyright holder for this preprint (which was not certified by peer review) is the author/funder, who has granted medRxiv a license to display the preprint in

All rights reserved. No reuse allowed without permission.

$\mathrm{API}^{69}$. All continuous variables were standardized using a z-score normalization pre-modelling, such that the reported effect sizes refer to the change in brain age delta associated with a one standard deviation increase in the given variable. For assessing the significance of the associations, we computed a Bonferroni-corrected p-value threshold $p_{\text {bonferroni }}=0.05 / 394=1.12 \times 10^{-4}$.

\section{Transfer learning to clinical samples}

In the transfer learning analysis we trained multiple binary models to predict whether a participant had a given diagnosis or belonged to the control group, based on various levels of information from the brain age model. For this purpose, we used the clinical dataset which was previously unseen by the model, and matched controls groups drawn from the external dataset used in the generalization test.

\section{Control matching}

To avoid biases in the case-control datasets we drew the subsets of controls independently for each scanner in the patient dataset, matching empirical distributions of age and sex in the corresponding case subset. For each disorder, for each scanner, the control group was created by drawing $n_{\text {case }}$ controls, without replacement, using the sampling procedure described for the post-hoc generalization analysis.

\section{Feature extraction}

To generate the feature vectors for each participant we used the trained SFCN-reg model as an encoder, up until and including the global average-pooling layer. Running a single MRI through the model up until this point results in a 64-dimensional vector representing the original image. Each of the dimensions $n=[0, \ldots, 63]$ in this space represent a high level feature of the brain, and each participant $X_{i}=\left[X_{i, 0}, \ldots, X_{i, 63}\right]$ is encoded as a point in this space. When used as a predictor in the subsequent modelling phase, each of these 64 dimensions were treated as an independent variable.

\section{Modelling}

For each disorder we compared three different LASSO models, all trained and evaluated using the following general procedure, but on different covariate sets. We first stratified the given dataset on disorder, age and sex, respectively, and split it into 5 folds. We performed an outer cross-validation over these splits to allow us to have an out-of-sample prediction for each participant. When training 
medRxiv preprint doi: https://doi.org/10.1101/2021.10.29.21265645; this version posted October 30, 2021. The copyright holder for this preprint (which was not certified by peer review) is the author/funder, who has granted medRxiv a license to display the preprint in

All rights reserved. No reuse allowed without permission.

a model on the training folds we performed an inner cross-validation to find the optimal value of the penalty parameter $\lambda$. The nested cross-validation procedure is illustrated in Supplementary Figure 5. Having found $\lambda$, we retrained the model on all the data from the four training folds. The models were implemented using sklearn's LogisticRegression ${ }^{70}$ with an $l_{1}$-penalty. Having the out-of-sample predictions for all the participants allowed us to calculate and compare AUCs based on the entire case-control dataset for the given disorder.

In addition to training the LASSO-model based on the brain age features, we trained an MLP using Keras with the same inputs. We did not optimize hyperparameters for this model, but observed similar results as the best LASSO models with the initial configuration (Supplementary Figure 6). The main benefit of the MLP is that it does not require a two-step process for the clinical prediction models, first processing the images with the encoder and then doing a prediction via a separate API, but can be implemented as an end-to-end binary classifier in Keras taking MRIs as inputs, and thus are more accessible for use by others.

\section{Data availability}

The raw data incorporated in this work were gathered from various resources. Material requests will need to be placed with individual principal investigators. A detailed overview of the independent datasets, and their origins, is provided in Supplementary Table 5.

\section{Code availability}

All of the trained brain age models, the end-to-end clinical predictors, and a pipeline for preprocessing images is released in our GitHub repo at http://www.github.com/estenhl/pyment-public

\section{Acknowledgements}

This work was funded by the UiO:LifeScience Convergence Environment (project: 4MENT), The Research Council of Norway (302854, 223273, 249795, 298646, 300767), the South-Eastern Norway Regional Health Authority (2014097, 2016083, 2018037, 2018076, 2019101), Stiftelsen Kristian Gerhard Jebsen, ERA-Net Cofund through the ERA PerMed project IMPLEMENT, and the European Research Council under the European Union's Horizon 2020 research and Innovation program (ERC StG, Grant 802998) and the Wellcome Trust grant (215698/Z/19/Z). T.W. gratefully acknowledges support from the European Unions Horizon 2020 research and innovation 
medRxiv preprint doi: https://doi.org/10.1101/2021.10.29.21265645; this version posted October 30, 2021. The copyright holder for this preprint (which was not certified by peer review) is the author/funder, who has granted medRxiv a license to display the preprint in All rights reserved. No reuse allowed without permission.

programme under the Marie Sklodowska-Curie grant agreement No 895011. A.F.M. gratefully acknowledges support from the European Research Council (ERC, grant number 10100118) and the Dutch Organisation for Scientific Research (016.156.415). Part of the computation was performed on the Norwegian high-performance computation resources, sigma2 (NN9767K/NS9769K). Data used in preparation of this article were obtained from the Alzheimers Disease Neuroimaging Initiative (ADNI) and the Australian Imaging Biomarkers and Lifestyle Study of Ageing (AIBL) databases (adni.loni.usc.edu), and the Pediatric Imaging, Neurocognition and Genetics (PING) study database (chd.ucsd.edu/research/ping-study.html, now shared through the NIMH Data Archive (NDA)). The investigators within the ADNI, AIBL, and PING studies contributed to the design and implementation of ADNI, AIBL, and PING or provided data but did not participate in the analysis or writing of this report. This publication is solely the responsibility of the authors and does not necessarily represent the views of the National Institutes of Health or PING investigators. 
medRxiv preprint doi: https://doi.org/10.1101/2021.10.29.21265645; this version posted October 30, 2021. The copyright holder for this preprint (which was not certified by peer review) is the author/funder, who has granted medRxiv a license to display the preprint in

All rights reserved. No reuse allowed without permission.

\section{References}

1. Stephan, B. C. M. \& Brayne, C. Assessing the risk of dementia in the aging population. Nat Rev Neurol 5, 417-418 (2009).

2. Thapar, A. \& Riglin, L. The importance of a developmental perspective in Psychiatry: what do recent genetic-epidemiological findings show? Mol Psychiatry 25, 1631-1639 (2020).

3. Franke, K., Ziegler, G., Klöppel, S. \& Gaser, C. Estimating the age of healthy subjects from T1-weighted MRI scans using kernel methods: Exploring the influence of various parameters. NeuroImage 50, 883-892 (2010).

4. Franke, K., Luders, E., May, A., Wilke, M. \& Gaser, C. Brain maturation: Predicting individual BrainAGE in children and adolescents using structural MRI. NeuroImage 63, 1305-1312 (2012).

5. Cole, J. H. \& Franke, K. Predicting Age Using Neuroimaging: Innovative Brain Ageing Biomarkers. Trends in Neurosciences 40, 681-690 (2017).

6. Cole, J. H. et al. Predicting brain age with deep learning from raw imaging data results in a reliable and heritable biomarker. NeuroImage 163, 115-124 (2017).

7. Franke, K. \& Gaser, C. Ten Years of BrainAGE as a Neuroimaging Biomarker of Brain Aging: What Insights Have We Gained? Front. Neurol. 10. (2021) (2019).

8. Kaufmann, T. et al. Common brain disorders are associated with heritable patterns of apparent aging of the brain. Nat Neurosci 22, 1617-1623 (2019).

9. Schnack, H. G. et al. Accelerated Brain Aging in Schizophrenia: A Longitudinal Pattern Recognition Study. AJP 173, 607-616 (2016).

10. Nenadić, I., Dietzek, M., Langbein, K., Sauer, H. \& Gaser, C. BrainAGE score indicates accelerated brain aging in schizophrenia, but not bipolar disorder. Psychiatry Research: Neuroimaging 266, 86-89 (2017).

11. Rokicki, J. et al. Multimodal imaging improves brain age prediction and reveals distinct abnormalities in patients with psychiatric and neurological disorders. Human Brain Mapping 42, $1714-1726(2021)$.

12. Han, L. K. M. et al. Brain aging in major depressive disorder: results from the ENIGMA major depressive disorder working group. Mol Psychiatry, 1-16 (2020).

13. Liem, F. et al. Predicting brain-age from multimodal imaging data captures cognitive impairment. NeuroImage 148, 179-188 (2017). 
medRxiv preprint doi: https://doi.org/10.1101/2021.10.29.21265645; this version posted October 30, 2021. The copyright holder for this preprint (which was not certified by peer review) is the author/funder, who has granted medRxiv a license to display the preprint in

All rights reserved. No reuse allowed without permission.

14. Elliott, M. L. et al. Brain-age in midlife is associated with accelerated biological aging and cognitive decline in a longitudinal birth cohort. Mol Psychiatry, 1-10 (2019).

15. Wang, J. et al. Gray Matter Age Prediction as a Biomarker for Risk of Dementia. PNAS 116, 21213-21218 (2019).

16. Gaser, C. et al. BrainAGE in Mild Cognitive Impaired Patients: Predicting the Conversion to Alzheimer's Disease. PLOS ONE 8, e67346 (2013).

17. Høgestøl, E. A. et al. Cross-Sectional and Longitudinal MRI Brain Scans Reveal Accelerated Brain Aging in Multiple Sclerosis. Front. Neurol. 0. (2021) (2019).

18. Smith, S. M., Vidaurre, D., Alfaro-Almagro, F., Nichols, T. E. \& Miller, K. L. Estimation of brain age delta from brain imaging. NeuroImage 200, 528-539 (2019).

19. Abrol, A. et al. Deep learning encodes robust discriminative neuroimaging representations to outperform standard machine learning. Nature Communications 12, 353 (2021).

20. Oquendo, M. A. et al. Machine learning and data mining: strategies for hypothesis generation. Mol Psychiatry 17, 956-959 (2012).

21. Peng, H., Gong, W., Beckmann, C. F., Vedaldi, A. \& Smith, S. M. Accurate brain age prediction with lightweight deep neural networks. Medical Image Analysis 68, 101871 (2021).

22. Gong, W., Beckmann, C. F., Vedaldi, A., Smith, S. M. \& Peng, H. Optimising a Simple Fully Convolutional Network for Accurate Brain Age Prediction in the PAC 2019 Challenge. Frontiers in Psychiatry 12, 658 (2021).

23. Dinsdale, N. K. et al. Learning patterns of the ageing brain in MRI using deep convolutional networks. NeuroImage 224, 117401 (2021).

24. Hofmann, S. M. et al. Towards the Interpretability of Deep Learning Models for Human Neuroimaging. bioRxiv, 2021.06.25.449906 (2021).

25. Arbabshirani, M. R., Plis, S., Sui, J. \& Calhoun, V. D. Single subject prediction of brain disorders in neuroimaging: Promises and pitfalls. NeuroImage. Individual Subject Prediction 145, 137-165 (2017).

26. Valverde, J. M. et al. Transfer Learning in Magnetic Resonance Brain Imaging: A Systematic Review. Journal of Imaging 7, 66 (2021).

27. Bengio, Y. Deep Learning of Representations for Unsupervised and Transfer Learning in Proceedings of ICML Workshop on Unsupervised and Transfer Learning (JMLR Workshop and Conference Proceedings, 2012), 17-36. (2021). 
medRxiv preprint doi: https://doi.org/10.1101/2021.10.29.21265645; this version posted October 30, 2021. The copyright holder for this preprint (which was not certified by peer review) is the author/funder, who has granted medRxiv a license to display the preprint in

All rights reserved. No reuse allowed without permission.

28. Sharif Razavian, A., Azizpour, H., Sullivan, J. \& Carlsson, S. CNN Features Off-the-Shelf: An Astounding Baseline for Recognition in (2014), 806-813. (2021).

29. Russakovsky, O. et al. ImageNet Large Scale Visual Recognition Challenge. Int J Comput Vis 115, 211-252 (2015).

30. Jonsson, B. A. et al. Brain age prediction using deep learning uncovers associated sequence variants. Nature Communications 10, 5409 (2019).

31. Lu, B. et al. A Practical Alzheimer Disease Classifier via Brain Imaging-Based Deep Learning on 85,721 Samples preprint (Neuroscience, 2020). (2021).

32. Bashyam, V. M. et al. MRI signatures of brain age and disease over the lifespan based on a deep brain network and 14468 individuals worldwide. Brain 143, 2312-2324 (2020).

33. Chen, S., Zhang, C., Dong, M., Le, J. \& Rao, M. Using Ranking-CNN for Age Estimation in 2017 IEEE Conference on Computer Vision and Pattern Recognition (CVPR) (IEEE, Honolulu, HI, 2017), 742-751. ISBN: 978-1-5386-0457-1. (2021).

34. He, S. et al. Multi-channel attention-fusion neural network for brain age estimation: Accuracy, generality, and interpretation with 16,705 healthy MRIs across lifespan. Medical Image Analysis 72, 102091 (2021).

35. Dockès, J., Varoquaux, G. \& Poline, J.-B. Preventing dataset shift from breaking machinelearning biomarkers. GigaScience 10. (2021) (2021).

36. Smith, S. M. \& Nichols, T. E. Statistical Challenges in "Big Data" Human Neuroimaging. Neuron 97, 263-268 (2018).

37. Alfaro-Almagro, F. et al. Confound modelling in UK Biobank brain imaging. NeuroImage 224, 117002 (2021).

38. Johnson, M. H. Functional brain development in humans. Nat Rev Neurosci 2, 475-483 (2001).

39. Fjell, A. M. \& Walhovd, K. B. Structural Brain Changes in Aging: Courses, Causes and Cognitive Consequences. Reviews in the Neurosciences 21, 187-222 (2010).

40. Ren, Y., Luo, Q., Gong, W. \& Lu, W. Transfer Learning Models on Brain Age Prediction in Proceedings of the Third International Symposium on Image Computing and Digital Medicine - ISICDM 2019 (ACM Press, Xi'an, China, 2019), 278-282. ISBN: 978-1-4503-7262-6. (2021).

41. Boyle, R. et al. Brain-predicted age difference score is related to specific cognitive functions: a multi-site replication analysis. Brain Imaging and Behavior 15, 327-345 (2021). 
medRxiv preprint doi: https://doi.org/10.1101/2021.10.29.21265645; this version posted October 30, 2021. The copyright holder for this preprint (which was not certified by peer review) is the author/funder, who has granted medRxiv a license to display the preprint in

All rights reserved. No reuse allowed without permission.

42. Butler, E. R. et al. Pitfalls in brain age analyses. Human Brain Mapping 42, 4092-4101 (2021).

43. De Lange, A.-M. G. \& Cole, J. H. Commentary: Correction procedures in brain-age prediction. Neuroimage Clin 26, 102229 (2020).

44. Dinsdale, N. K., Jenkinson, M. \& Namburete, A. I. L. Unlearning Scanner Bias for MRI Harmonisation in Medical Image Computing and Computer Assisted Intervention - MICCAI 2020 (eds Martel, A. L. et al.) (Springer International Publishing, Cham, 2020), 369-378. ISBN: 978-3-030-59713-9.

45. Karasik, D., Demissie, S., Cupples, L. A. \& Kiel, D. P. Disentangling the Genetic Determinants of Human Aging: Biological Age as an Alternative to the Use of Survival Measures. The Journals of Gerontology: Series A 60, 574-587 (2005).

46. Ashpole, N. M., Sanders, J. E., Hodges, E. L., Yan, H. \& Sonntag, W. E. Growth hormone, insulin-like growth factor-1 and the aging brain. Experimental Gerontology. Proceedings of the Twelfth International Symposium on the Neurobiology and Neuroendocrinology of Aging, Bregenz, Austria July 27-August 1, 2014 68, 76-81 (2015).

47. Roth, J. et al. HbA1c and Age in Non-Diabetic Subjects: An Ignored Association? Exp Clin Endocrinol Diabetes 124, 637-642 (2016).

48. Gamaldo, A. A., Ferrucci, L., Rifkind, J., Longo, D. L. \& Zonderman, A. B. Relationship Between Mean Corpuscular Volume and Cognitive Performance in Older Adults. Journal of the American Geriatrics Society 61, 84-89 (2013).

49. Cole, J. H. Multimodality neuroimaging brain-age in UK biobank: relationship to biomedical, lifestyle, and cognitive factors. Neurobiology of Aging 92, 34-42 (2020).

50. De Lange, A.-M. G. et al. Multimodal brain-age prediction and cardiovascular risk: The Whitehall II MRI sub-study. NeuroImage 222, 117292 (2020).

51. Wrigglesworth, J. et al. Factors associated with brain ageing - a systematic review. $B M C$ Neurology 21, 312 (2021).

52. Beck, D. et al. Cardiometabolic risk factors associated with brain age and accelerate brain ageing. Hum Brain Mapp (2021).

53. Franke, K., Gaser, C., Manor, B. \& Novak, V. Advanced BrainAGE in older adults with type 2 diabetes mellitus. Front. Aging Neurosci. 5. (2021) (2013). 
medRxiv preprint doi: https://doi.org/10.1101/2021.10.29.21265645; this version posted October 30, 2021. The copyright holder for this preprint (which was not certified by peer review) is the author/funder, who has granted medRxiv a license to display the preprint in

All rights reserved. No reuse allowed without permission.

54. Smith, S. M. et al. Brain aging comprises many modes of structural and functional change with distinct genetic and biophysical associations. eLife 9 (eds Peelle, J. E., de Lange, F. P., Madan, C. \& Nyberg, L.) e52677 (2020).

55. Hahn, T. et al. From 'loose fitting' to high-performance, uncertainty-aware brain-age modelling. Brain 144, e31-e31 (2021).

56. Bashyam, V., Shou, H. \& Davatzikos, C. Reply: From 'loose fitting' to high-performance, uncertainty-aware brain-age modelling. Brain 144, e32-e32 (2021).

57. Thompson, P. M. et al. ENIGMA and global neuroscience: A decade of large-scale studies of the brain in health and disease across more than 40 countries. Transl Psychiatry 10, 1-28 (2020).

58. Richard, G. et al. Assessing distinct patterns of cognitive aging using tissue-specific brain age prediction based on diffusion tensor imaging and brain morphometry. PeerJ 6, e5908 (2018).

59. Vidal-Piñeiro, D. et al. Individual variations in "Brain age" relate to early life factors more than to longitudinal brain change. bioRxiv, 2021.02.08.428915 (2021).

60. Ségonne, F. et al. A hybrid approach to the skull stripping problem in MRI. Neuroimage 22, 1060-1075 (2004).

61. Jenkinson, M., Beckmann, C. F., Behrens, T. E. J., Woolrich, M. W. \& Smith, S. M. FSL. NeuroImage. 20 YEARS OF fMRI 62, 782-790 (2012).

62. Jenkinson, M. \& Smith, S. A global optimisation method for robust affine registration of brain images. Med Image Anal 5, 143-156 (2001).

63. Simonyan, K. \& Zisserman, A. Very Deep Convolutional Networks for Large-Scale Image Recognition. arXiv:1409.1556 [cs]. (2021) (2015).

64. Xia, T., Chartsias, A., Wang, C. \& Tsaftaris, S. A. Learning to synthesise the ageing brain without longitudinal data. Medical Image Analysis 73, 102169 (2021).

65. Chollet, F. et al. Keras 2015.

66. Abadi, M. et al. TensorFlow: Large-Scale Machine Learning on Heterogeneous Distributed Systems, 19 .

67. Smith, L. N. Cyclical Learning Rates for Training Neural Networks. arXiv:1506.01186 [cs]. (2021) (2017). 
medRxiv preprint doi: https://doi.org/10.1101/2021.10.29.21265645; this version posted October 30, 2021. The copyright holder for this preprint (which was not certified by peer review) is the author/funder, who has granted medRxiv a license to display the preprint in All rights reserved. No reuse allowed without permission.

68. Millard, L. A., Davies, N. M., Gaunt, T. R., Davey Smith, G. \& Tilling, K. Software Application Profile: PHESANT: a tool for performing automated phenome scans in UK Biobank. International Journal of Epidemiology 47, 29-35 (2018).

69. Seabold, S. \& Perktold, J. Statsmodels: Econometric and Statistical Modeling with Python in (Austin, Texas, 2010), 92-96. (2021).

70. Pedregosa, F. et al. Scikit-learn: Machine Learning in Python. MACHINE LEARNING IN PYTHON, 6 . 